\title{
Desarrollo de estrategias de evaluación de competencias transversales en asignaturas de ingeniería mecánica y de materiales
}

Javier Carballeira Morado, José Martínez Casas, Óscar Sahuquillo Navarro, Águeda Sonseca Olalla, Francisco David Denia Guzmán, Josep Lluís Suñer Martínez, Paloma Vila Tortosa, Juan José Ródenas García y Onofre Marco Alacid

Departamento de Ingeniería Mecánica y de Materiales - jacarmo@mcm.upv.es

\begin{abstract}
The international accreditation for the Master and Bachelor degrees offered at our university, together with the demands of the employers, have made it clear that students' curricula should specify not only what they have studied, but also what they are actually able to do. This work presents the results obtained within the frame of an innovative project (UPV - PIME program) on the evaluation of three generic competences that have been traditionally worked in subjects of mechanical and materials engineering: capacity for problem solving; capacity for applying knowledge in practice; and communication skills (both oral and written). Different tools for the assessment of these competences have developed with two main objectives: first, to quantify the level of achievement of the students in order to give a numerical evaluation; and second, to be used by the students as a learning material so that they can improve their capacities. These tools are based on the observation of some learning outcomes associated to these competences. Some evaluation activities have been proposed within the different subjects that allow to assess not only the specific scientific-technical competences, but also some of the generic ones.
\end{abstract}

Keywords: assessment; generic competences. 
Desarrollo de estrategias de evaluación de competencias transversales en asignaturas de ingeniería mecánica y de materiales

\begin{abstract}
Resumen
La acreditación internacional de los títulos de Grado y Máster en nuestra universidad, junto con las demandas de los empleadores, han puesto en evidencia que los planes de estudio deben especificar no sólo lo que se ha estudiado, sino también lo que se es capaz de hacer realmente. Este trabajo presenta los resultados obtenidos en el marco de un proyecto de innovación docente (programa PIME) sobre la evaluación de tres competencias transversales que se han trabajado tradicionalmente en asignaturas de ingeniería mecánica y materiales: la capacidad para la resolución de problemas; la capacidad para aplicar los conocimientos a la práctica; y habilidades de comunicación (oral y escrita). Se han desarrollado diferentes herramientas para la evaluación de estas competencias con dos objetivos principales: en primer lugar, para cuantificar el nivel de logro de los estudiantes con el fin de dar una evaluación numérica; y en segundo, como material de aprendizaje para que los estudiantes puedan mejorar sus capacidades. Estas herramientas se basan en la observación de algunos resultados de aprendizaje asociados a estas competencias. Se han propuesto algunas actividades de evaluación en las diferentes asignaturas que permiten evaluar no sólo las competencias científico-técnicas específicas, sino también algunas de las transversales.
\end{abstract}

Palabras clave: evaluación, competencias transversales.

\title{
Introducción
}

Los planes de estudio de Grado y de Máster impartidos en nuestra universidad y desarrollados en el marco del Espacio Europeo de Educación Superior están basados en una aproximación por competencias (Sursock, 2010; Murias, 2007; Rieckmann, 2012). En estos planes de estudio se detallan las competencias específicas y transversales que se van a trabajar en el título, e incluso, en qué asignaturas a lo largo del mismo. La evaluación de las competencias específicas se documenta en el expediente académico mediante una valoración numérica, pero el nivel de desarrollo alcanzado en las competencias transversales no aparece. Se sobreentiende en cierta medida que se han trabajado de forma satisfactoria a lo largo de los cursos.

La acreditación internacional de los títulos, junto con las demandas de los empleadores para disponer de una información más precisa acerca de las capacidades de los egresados, han impulsado a la universidad a resolver esta carencia (Andrews, 2008; Entwistle, 2004). En 
Javier Carballeira Morado, José Martínez Casas, Óscar Sahuquillo Navarro, Águeda Sonseca Olalla, Francisco David Denia Guzmán, Josep Lluís Suñer Martínez, Paloma Vila Tortosa, Juan José Ródenas García y Onofre Marco Alacid

concreto, la Universitat Politècnica de València, a la que pertenecen los autores de este trabajo, ha puesto en marcha un proyecto institucional en esta línea (UPV, 2014) y ha iniciado una serie de acciones para favorecer y financiar iniciativas innovadoras en este sentido. Entre ellas, incluir de forma preferente la evaluación de competencias transversales dentro del programa anual de Proyectos de Innovación y Mejora Educativa (PIME), dentro del que se enmarca este trabajo.

De este modo, en este trabajo se resume el trabajo desarrollado y los resultados obtenidos en un proyecto de innovación y mejora educativa aplicado en varias asignaturas del Departamento de Ingeniería Mecánica y de Materiales en diferentes títulos de la Universitat Politècnica de València. Se han involucrado asignaturas sobre Teoría De Máquinas y Mecanismos, Integridad Estructural, Vibraciones y Materiales del Máster Universitario en Ingeniería Aeronáutica y del Grado en Ingeniería Mecánica de la Escuela Técnica Superior de Ingeniería del Diseño; así como del Grado en Ingeniería Química, del Grado en Ingeniería en Organización Industrial, y del Grado en Ingeniería en Tecnologías Industriales de la Escuela Técnica Superior de Ingenieros Industriales. Disponer de un número alto de asignaturas de diferentes niveles era interesante para poder validar las herramientas desarrolladas comparando los resultados obtenidos en cada una. De forma que los estudiantes de Máster puntuaran más alto que los estudiantes de Grado, en general, y con valores acorde a los resultados de aprendizaje esperados (UPV, 2014).

En primer lugar se explicarán los objetivos que impulsaron el proyecto, para a continuación describir el desarrollo de la innovación planteada. Se explicará el proceso seguido para generar una serie de instrumentos de evaluación para la cuantificación del nivel de desarrollo de las competencias transversales implicadas en el proyecto. En los Resultados se mostrará un ejemplo aplicado a una asignatura. Finalmente, se comentarán las conclusiones más relevantes de la experiencia adquirida y su importancia de cara al futuro.

\section{Objetivos}

El objetivo principal de este proyecto era desarrollar y experimentar instrumentos de evaluación que permitieran cuantificar el nivel de desarrollo de los estudiantes en las competencias: Aplicación y pensamiento práctico, Análisis y Resolución de Problemas, y Comunicación Efectiva, según la denominación empleada en nuestra universidad (UPV, 2014), que se trabajan empleando diferentes metodologías en cada asignatura. Nace del interés de varios profesores del Departamento de Ingeniería Mecánica y de Materiales en la necesidad de evaluar de una forma objetiva las competencias transversales. Motivados por el proyecto institucional, por un lado, y por la posibilidad de disponer de herramientas de evaluación en sus asignaturas que fueran además herramientas de aprendizaje para los 
Desarrollo de estrategias de evaluación de competencias transversales en asignaturas de ingeniería mecánica y de materiales

alumnos. En la línea del Assessment for Learning (AfL), Evaluación para el Aprendizaje (McDowell, 2011), el equipo consideraba muy interesante la idea de desarrollar actividades y herramientas de evaluación que permitieran, no sólo calificar numéricamente el nivel de desempeño de los estudiantes en las competencias científico-técnicas específicas, sino además, cuantificar el nivel de logro en las competencias transversales y servir como herramientas de aprendizaje a los estudiantes para mejorar su desarrollo.

\section{Desarrollo de la innovación}

El trabajo desarrollado a lo largo del proyecto se puede esquematizar de forma cronológica como:

1. Análisis de los resultados de aprendizaje asociados a cada competencia transversal del proyecto y establecidos desde la universidad (UPV, 2014).

2. Determinación de niveles de desarrollo de los resultados de aprendizaje según el nivel de los estudios (Grado y Máster).

3. Búsqueda de evidencias e indicadores que permitieran valorar el grado de desarrollo de la competencia.

4. Generación de instrumentos de evaluación para la observación y cuantificación de estos indicadores en las actividades de evaluación desarrolladas en cada asignatura.

5. Propuesta de nuevas actividades de evaluación que faciliten la puesta en marcha de las competencias transversales por parte de los estudiantes, además de involucrar la aplicación de las competencias específicas.

Mientras la definición de la competencia de Comunicación Efectiva, tanto oral como escrita, era clara para todos los componentes del equipo, las diferencias entre las competencias de Análisis y Resolución de Problemas y de Aplicación y Pensamiento Práctico, no lo eran. En una primera fase de reuniones y discusión, se intentó establecer el alcance de cada una a partir de las definiciones empleadas por la universidad, para tener claro que evidencias e indicadores reflejarían el dominio de éstas. Se llegó a la conclusión, de que la competencia en Análisis y Resolución de Problemas se refiere al proceso de solución de un problema una vez planteado, mientras la competencia en Aplicación y Pensamiento Práctico sería más bien la capacidad para plantear de forma adecuada un problema desde una situación real (Deusto, 2014).

En concreto, se definió la competencia en Análisis y Resolución de Problemas como la capacidad para analizar y resolver un problema de forma eficaz. Se entiende por problema aquel ejercicio en el que se plantea una situación nueva y abierta a los estudiantes, con más de una solución posible y que se puede resolver desde varias aproximaciones distintas 
Javier Carballeira Morado, José Martínez Casas, Óscar Sahuquillo Navarro, Águeda Sonseca Olalla, Francisco David Denia Guzmán, Josep Lluís Suñer Martínez, Paloma Vila Tortosa, Juan José Ródenas García y Onofre Marco Alacid

(Pozo, 2009). De este modo, esta competencia se puede descomponer en tres resultados de aprendizaje: identificar un problema real y determinar sus partes principales; aplicar los métodos aprendidos para analizar el problema, extraer la información más relevante y proponer distintas alternativas de solución; usar la experiencia y juicio crítico para evaluar y generar una solución efectiva y eficiente.

La competencia en Aplicación y Pensamiento Práctico se define como la capacidad para identificar los objetivos a alcanzar para resolver una situación real y establecer un plan apropiado para ello, teniendo en cuenta las restricciones, recursos e información disponibles. Se trata de desarrollar un modo de pensar dirigido a la acción que permite adaptarse a nuevas situaciones, tomando las decisiones necesarias para salvar las incertidumbres que aparecen en una situación real, alejada de los ejercicios académicos.

Para las tres competencias se planteó una aproximación similar a la empleada en el Marco Común Europeo de Referencia para las Lenguas: Aprendizaje, Enseñanza, Evaluación (MECD, 2002). Se han establecido escalas de desarrollo para cada competencia con 6 niveles de dominio, análogos a los niveles A1 hasta C2. Se acordó que los cuatro primeros corresponderían a los resultados de aprendizaje de los estudios de Grado, y se reservarían los dos últimos para los resultados de aprendizaje de los estudios de Máster.

Simultáneamente, se buscaron aquellos indicadores y evidencias que permitieran observar el grado de desarrollo de los resultados de aprendizaje, y así cuantificar el nivel del estudiante en la competencia. Las Tablas 1 y 2 presentan las escalas de desarrollo para las competencias en Análisis y Resolución de Problemas, y en Aplicación y Pensamiento Práctico (las de Comunicación Efectiva son similares, pero se omiten por falta de espacio), que resumen el trabajo llevado a cabo en los puntos 1, 2 y 3 del esquema anterior.

Una vez establecidas las escalas, se han generado las herramientas de evaluación con el doble objetivo explicado anteriormente: evaluar las competencias transversales, y servir como material de aprendizaje a los estudiantes. Para las competencias en Análisis y Resolución de Problemas, y Aplicación y Pensamiento Práctico, se han planteado listas de control (Tablas 3 y 4, respectivamente). Para la competencia en Comunicación Efectiva, se presentan rúbricas para evaluar la competencia escrita (Tabla 5) y oral (Tabla 6).

A partir de la experiencia obtenida en las primeras pruebas realizadas con estas herramientas, en la última fase del proyecto se han propuesto actividades de evaluación más completas, para este curso o el siguiente, atendiendo a los objetivos planteados. Además, se han adaptado los instrumentos de evaluación a las asignaturas para facilitar su utilización. En el siguiente apartado de Resultados se muestra un caso práctico de una de las asignaturas involucradas en el proyecto.

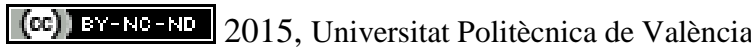

Congreso In-Red (2015) 
Desarrollo de estrategias de evaluación de competencias transversales en asignaturas de ingeniería mecánica y de materiales

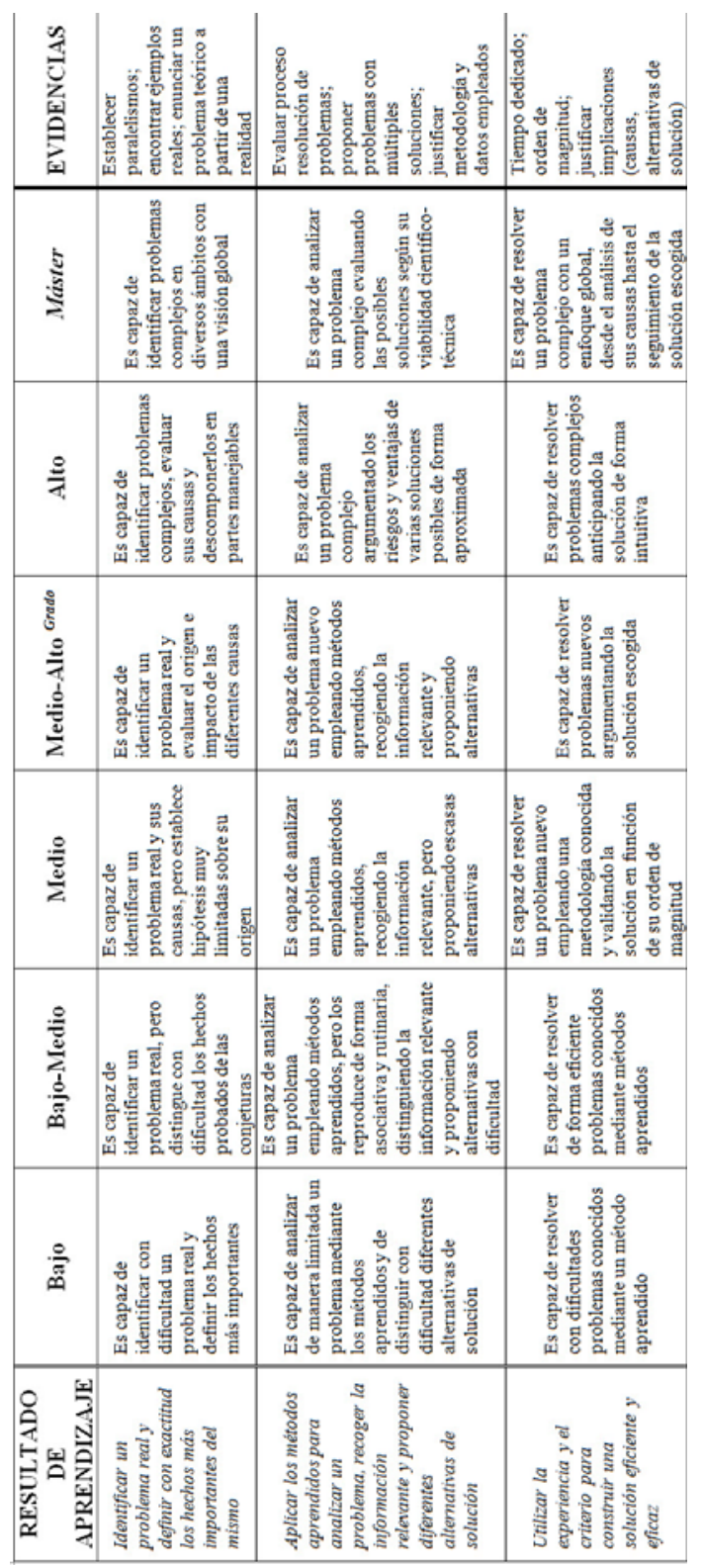

Tabla 1. Escala de desarrollo e indicadores para los resultados de aprendizaje de la competencia en Análisis y Resolución de Problemas

(c)) EY-NC-ND 2015, Universitat Politècnica de València 
Javier Carballeira Morado, José Martínez Casas, Óscar Sahuquillo Navarro, Águeda Sonseca Olalla, Francisco David Denia Guzmán, Josep Lluís Suñer Martínez, Paloma Vila Tortosa, Juan José Ródenas García y Onofre Marco Alacid

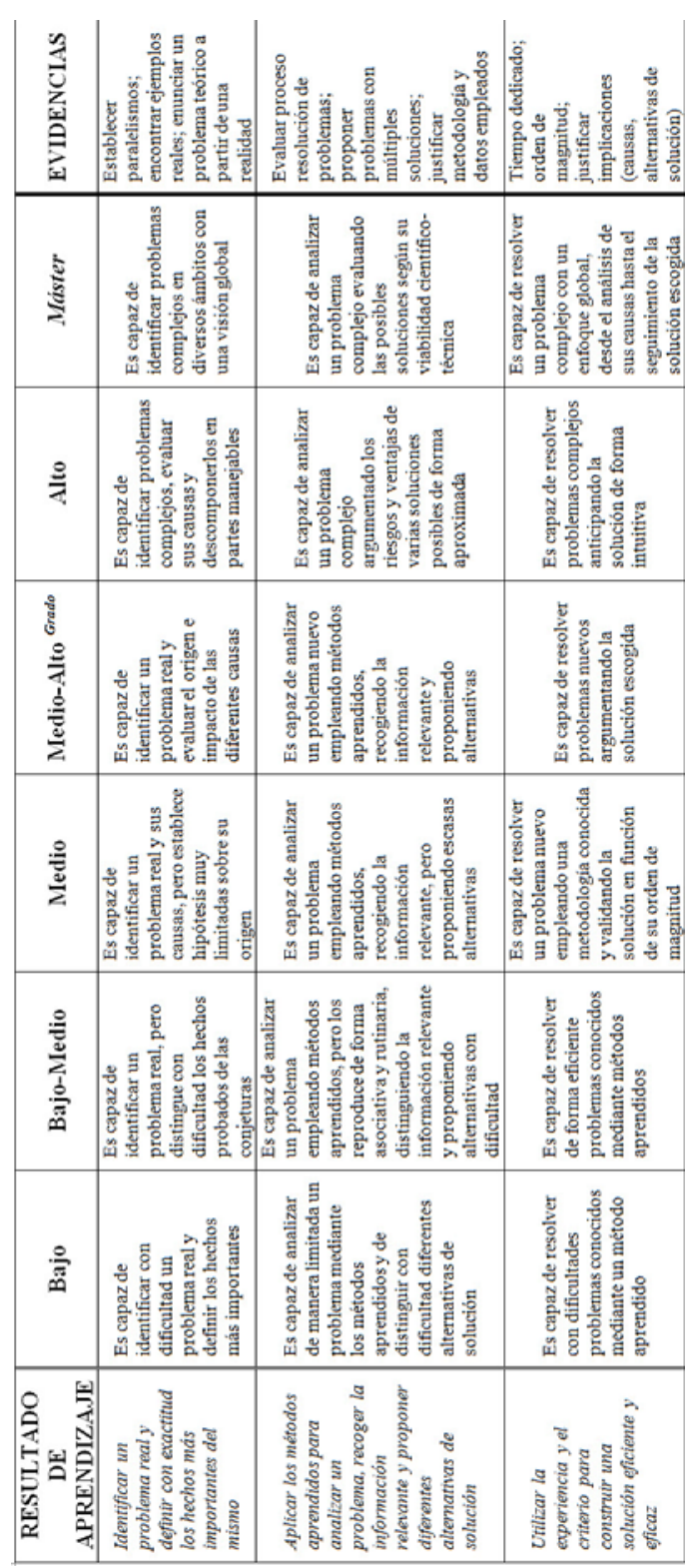

Tabla 2. Escala de desarrollo e indicadores para los resultados de aprendizaje de la competencia en Aplicación y Pensamiento Práctico

(c)) EY-NC-ND 2015, Universitat Politècnica de València

Congreso In-Red (2015) 
Desarrollo de estrategias de evaluación de competencias transversales en asignaturas de ingeniería mecánica y de materiales

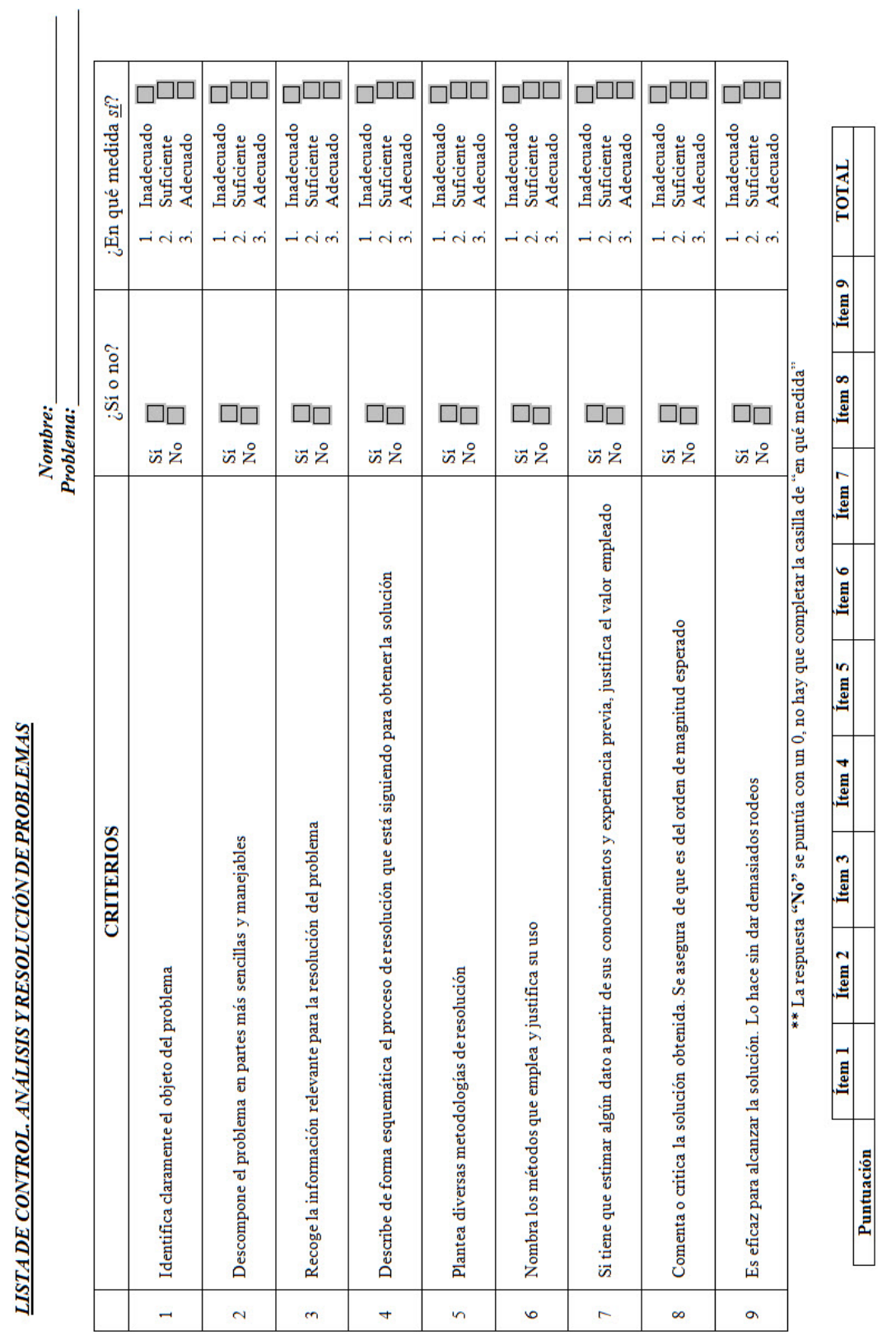

Tabla 3. Lista de control para la evaluación de la competencia en Análisis y Resolución de Problemas

(cc) EY-NC-ND 2015, Universitat Politècnica de València 
Javier Carballeira Morado, José Martínez Casas, Óscar Sahuquillo Navarro, Águeda Sonseca Olalla, Francisco David Denia Guzmán, Josep Lluís Suñer Martínez, Paloma Vila Tortosa, Juan José Ródenas García y Onofre Marco Alacid

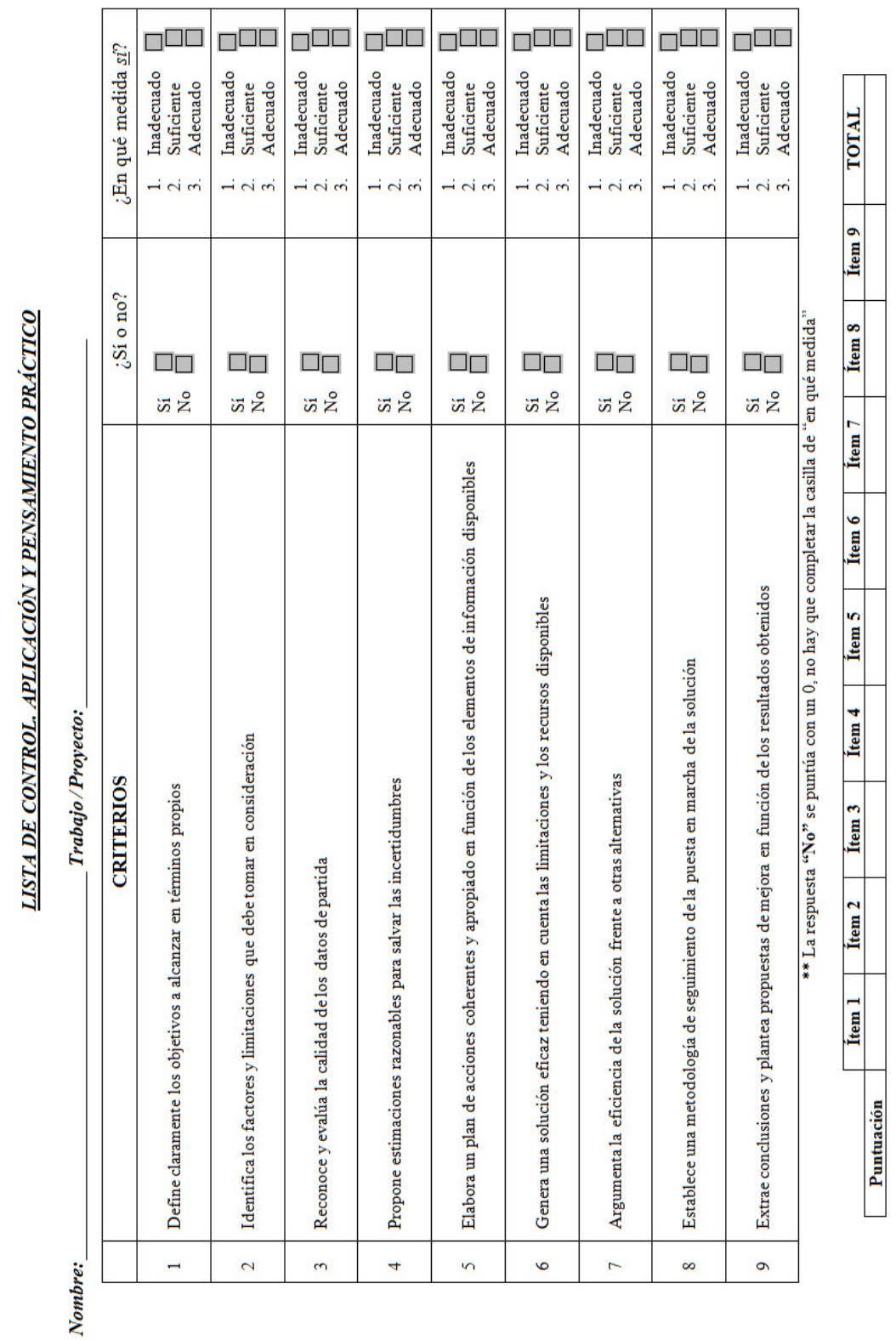

Tabla 4. Lista de control para la evaluación de la competencia en Aplicación y Pensamiento Práctico

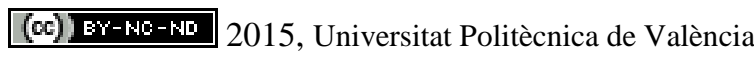

Congreso In-Red (2015) 
Desarrollo de estrategias de evaluación de competencias transversales en asignaturas de ingeniería mecánica y de materiales

\section{RÚBRICA COMUNICACIÓN ESCRITA}

\begin{tabular}{|c|c|c|c|c|c|c|c|}
\hline & & & & & $\begin{array}{l}\text { Nombre: } \\
\text { Problema: }\end{array}$ & & \\
\hline & & 0-No cumple & 1-Deficiente & 2-Regular & 3-Bueno & 4-Muy bueno & 5-Excelente \\
\hline & CRITERIOS & & & & & & \\
\hline 1 & $\begin{array}{l}\text { Realiza una } \\
\text { introducción } \\
\text { efectiva al tema }\end{array}$ & No la realiza & $\begin{array}{l}\text { Realiza introducción } \\
\text { mayoritariamente } \\
\text { incompleta }\end{array}$ & $\begin{array}{l}\text { Realiza introducción } \\
\text { incompleta }\end{array}$ & Realiza introducción & $\begin{array}{l}\text { Realiza introducción } \\
\text { y pone en situación } \\
\text { a los lectores }\end{array}$ & $\begin{array}{l}\text { Realiza introducción } \\
\text { indicando ejemplos } \\
\text { del interés del tema }\end{array}$ \\
\hline 2 & $\begin{array}{l}\text { Identifica los } \\
\text { objetivos e ideas } \\
\text { principales }\end{array}$ & No lo realiza & $\begin{array}{l}\text { Seflala alguno de los } \\
\text { objetivos }\end{array}$ & $\begin{array}{l}\text { Sertala los objetivos } \\
\text { de forma incompleta }\end{array}$ & $\begin{array}{l}\text { Señala todos los } \\
\text { objetivos }\end{array}$ & $\begin{array}{l}\text { Señala todos los } \\
\text { objetivos de forma } \\
\text { organizada y } \\
\text { sintética }\end{array}$ & $\begin{array}{l}\text { Señala todos los } \\
\text { objetivos de forma } \\
\text { organizada y } \\
\text { sintética, } \\
\text { relacionánđolos con } \\
\text { situaciones reales }\end{array}$ \\
\hline 3 & $\begin{array}{l}\text { Los resultados se } \\
\text { presentan } \\
\text { fundamentados } \\
\text { adecuadamente }\end{array}$ & No presenta & $\begin{array}{l}\text { Presenta algún } \\
\text { resultado }\end{array}$ & $\begin{array}{l}\text { Presenta de forma } \\
\text { incompleta }\end{array}$ & $\begin{array}{l}\text { Presenta todos los } \\
\text { resultados }\end{array}$ & $\begin{array}{l}\text { Presenta los } \\
\text { resultados } \\
\text { relevantes }\end{array}$ & $\begin{array}{l}\text { Presenta los } \\
\text { resultados } \\
\text { relevantes y los } \\
\text { fundamenta }\end{array}$ \\
\hline 4 & $\begin{array}{l}\text { Las conclusiones } \\
\text { son apropiadas y } \\
\text { sintéticas }\end{array}$ & No las realiza & $\begin{array}{l}\text { Seflala alguna } \\
\text { conclusión } \\
\text { incompleta }\end{array}$ & $\begin{array}{l}\text { Señala todas las } \\
\text { conclusiones de } \\
\text { forma incompleta }\end{array}$ & $\begin{array}{l}\text { Señala todas las } \\
\text { conclusiones }\end{array}$ & $\begin{array}{l}\text { Señala las } \\
\text { conclusiones más } \\
\text { relevantes de forma } \\
\text { organizada y } \\
\text { sintética }\end{array}$ & $\begin{array}{l}\text { Señala las } \\
\text { conclusiones más } \\
\text { relevantes de forma } \\
\text { organizada, sintética } \\
\text { y evalúa/analiza sus } \\
\text { consecuencias }\end{array}$ \\
\hline 5 & $\begin{array}{l}\text { Informe } \\
\text { estructurado y } \\
\text { coherente }\end{array}$ & No estructurado & $\begin{array}{l}\text { Estructura } \\
\text { incoherente }\end{array}$ & $\begin{array}{l}\text { Estructura coherente } \\
\text { pero incompleta }\end{array}$ & Estructura coherente & $\begin{array}{l}\text { Estructura } \\
\text { coherente, relación } \\
\text { entre secciones }\end{array}$ & $\begin{array}{l}\text { Estructura } \\
\text { coherente, relación } \\
\text { entre secciones y } \\
\text { orden justificado }\end{array}$ \\
\hline 6 & $\begin{array}{l}\text { Formato y estilo } \\
\text { profesional }\end{array}$ & No lo realiza & $\begin{array}{l}\text { Formato y estilo } \\
\text { inconsistente }\end{array}$ & $\begin{array}{l}\text { Formato y estilo } \\
\text { consistente pero } \\
\text { limitado }\end{array}$ & $\begin{array}{l}\text { Formato y estilo } \\
\text { correcto }\end{array}$ & $\begin{array}{l}\text { Formato y estilo } \\
\text { avanzado }\end{array}$ & $\begin{array}{l}\text { Formato y estilo } \\
\text { profesional }\end{array}$ \\
\hline 7 & Ortografia & $\begin{array}{l}\text { Carencia total de } \\
\text { ortografia }\end{array}$ & $\begin{array}{l}\text { Elevadas faltas de } \\
\text { ortografia }\end{array}$ & $\begin{array}{l}\text { Alguna falta de } \\
\text { ortografia presente }\end{array}$ & $\begin{array}{l}\text { Redacción sin faltas } \\
\text { de ortografia }\end{array}$ & $\begin{array}{l}\text { Ortografia correcta } \\
\text { y técnica }\end{array}$ & $\begin{array}{l}\text { Ortografia correcta, } \\
\text { técnica y apreciado } \\
\text { uso de sinónimos }\end{array}$ \\
\hline 8 & $\begin{array}{l}\text { Utiliza un lenguaje } \\
\text { técnico adecuado }\end{array}$ & $\begin{array}{l}\text { Lenguaje } \\
\text { inadecuado }\end{array}$ & $\begin{array}{l}\text { Lo usa con errores } \\
\text { reiterados }\end{array}$ & $\begin{array}{l}\text { Lo usa con alguin } \\
\text { error }\end{array}$ & $\begin{array}{l}\text { Lo utiliza } \\
\text { ocasionalmente }\end{array}$ & $\begin{array}{l}\text { Lo utiliza habitualy } \\
\text { adecuadamente }\end{array}$ & $\begin{array}{l}\text { Lo utiliza habitualy } \\
\text { adecuadamente, } e \\
\text { introduce nuevos } \\
\text { conceptos }\end{array}$ \\
\hline 9 & $\begin{array}{l}\text { Interpreta, } \\
\text { argumenta y } \\
\text { justifica la } \\
\text { información }\end{array}$ & No lo realiza & $\begin{array}{l}\text { Lo realiza de } \\
\text { manera errónea }\end{array}$ & $\begin{array}{l}\text { Lo realiza bien pero } \\
\text { de manera muy } \\
\text { limitada }\end{array}$ & $\begin{array}{l}\text { Lo realiza bien pero } \\
\text { aún incompleto }\end{array}$ & $\begin{array}{l}\text { Lo realiza de } \\
\text { manera correcta }\end{array}$ & $\begin{array}{l}\text { Lo realiza } \\
\text { correctamente, y } \\
\text { además con el } \\
\text { énfasis necesario }\end{array}$ \\
\hline 10 & $\begin{array}{l}\text { Elabora y utiliza } \\
\text { recursos gráficos } \\
\text { efectivos que dan } \\
\text { calidad al informe }\end{array}$ & $\begin{array}{l}\text { No utiliza recurso } \\
\text { alguno }\end{array}$ & $\begin{array}{l}\text { Utiliza recursos de } \\
\text { forma inadecuada }\end{array}$ & $\begin{array}{l}\text { Utiliza recursos } \\
\text { pero no aportan } \\
\text { calidad }\end{array}$ & $\begin{array}{l}\text { Utiliza recursos que } \\
\text { aportan calidad, } \\
\text { pero limitados }\end{array}$ & $\begin{array}{l}\text { Utiliza recursos que } \\
\text { aportan calidad }\end{array}$ & $\begin{array}{l}\text { Utiliza recursos } \\
\text { convencionales e } \\
\text { introduce nuevos } \\
\text { que dan } \\
\text { profesionalidad }\end{array}$ \\
\hline
\end{tabular}

\section{Notas adicionales}

Los diferentes ítems de la Rúbrica nos permiten evaluar los siguientes aspectos de la comunicación escrita, englobados en los resultados de aprendizaje de la competencia en cuestión:

- Items 1-4: Contenido Item 5: Estructura Item 6: Formato y estilo fitem 7: Ortografia ftem 8: Vocabulario

- Item 9: Interpretacióny justificación de la información Item 10: Recursos gráficos

\begin{tabular}{|c|c|c|c|c|c|c|c|c|}
\cline { 2 - 7 } \multicolumn{1}{c|}{} & İtem 1-4 & İtem 5 & İtem 6 & İtem 7 & İtem 8 & İtem 9 & İtem 10 & TOTAL \\
\hline Puntuación & & & & & & & & \\
\hline
\end{tabular}

Tabla 5. Rúbrica para la evaluación de la competencia en Comunicación Efectiva escrita

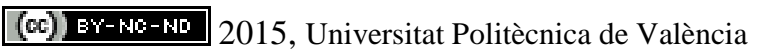

Congreso IN-RED (2015) 
Javier Carballeira Morado, José Martínez Casas, Óscar Sahuquillo Navarro, Águeda Sonseca Olalla, Francisco David Denia Guzmán, Josep Lluís Suñer Martínez, Paloma Vila Tortosa, Juan José Ródenas García y Onofre Marco Alacid

RÚBRICA COMUNICACIÓN ORAL

Nombre:

Expone:

Grupo:

Práctica:

\begin{tabular}{|c|c|c|c|c|c|c|c|}
\hline & CRITERIOS & 0 -No cumple & 1-Deficiente & 2-Regular & 3-Bueno & 4-Muy bueno & 5-Excelente \\
\hline 1 & $\begin{array}{l}\text { Realiza una } \\
\text { introducción } \\
\text { efectiva al tema }\end{array}$ & no la realiza & $\begin{array}{l}\text { realiza } \\
\text { introducción } \\
\text { mayoritariamente } \\
\text { incompleta }\end{array}$ & $\begin{array}{l}\text { realiza } \\
\text { introducción } \\
\text { incompleta }\end{array}$ & $\begin{array}{l}\text { realiza } \\
\text { introducción }\end{array}$ & $\begin{array}{l}\text { realiza } \\
\text { introducción y } \\
\text { pone en situación a } \\
\text { la audiencia }\end{array}$ & $\begin{array}{l}\text { realiza } \\
\text { introducción } \\
\text { indicando ejemplos } \\
\text { del interés del tema }\end{array}$ \\
\hline 2 & $\begin{array}{l}\text { Señala los objetivos } \\
\text { e ideas principales }\end{array}$ & no lo realiza & $\begin{array}{l}\text { señala alguno de } \\
\text { los objetivos }\end{array}$ & $\begin{array}{l}\text { señala los objetivos } \\
\text { de forma } \\
\text { incompleta }\end{array}$ & $\begin{array}{l}\text { señala todos los } \\
\text { objetivos }\end{array}$ & $\begin{array}{l}\text { señala todos los } \\
\text { objetivos de forma } \\
\text { organizada y } \\
\text { sintética }\end{array}$ & $\begin{array}{l}\text { señala todos los } \\
\text { objetivos de forma } \\
\text { organizada y } \\
\text { sintética, con un } \\
\text { enfoque aplicado }\end{array}$ \\
\hline 3 & $\begin{array}{l}\text { Presenta resultados } \\
\text { fundamentados } \\
\text { adecuadamente }\end{array}$ & no presenta & $\begin{array}{l}\text { presenta algún } \\
\text { resultado }\end{array}$ & $\begin{array}{l}\text { presenta de forma } \\
\text { incompleta }\end{array}$ & $\begin{array}{l}\text { presenta todos los } \\
\text { resultados }\end{array}$ & $\begin{array}{l}\text { presenta los } \\
\text { resultados } \\
\text { relevantes }\end{array}$ & $\begin{array}{l}\text { presenta los } \\
\text { resultados } \\
\text { relevantes y los } \\
\text { fundamenta }\end{array}$ \\
\hline 4 & $\begin{array}{l}\text { Conclusiones } \\
\text { apropiadas y } \\
\text { sintéticas }\end{array}$ & no las realiza & $\begin{array}{l}\text { señala alguna } \\
\text { conclusión }\end{array}$ & $\begin{array}{l}\text { señala } \\
\text { conclusiones de } \\
\text { forma incompleta }\end{array}$ & $\begin{array}{l}\text { señala todas las } \\
\text { conclusiones }\end{array}$ & $\begin{array}{l}\text { señala las } \\
\text { conclusiones más } \\
\text { relevantes } \\
\text { organizada y } \\
\text { sintéticamente }\end{array}$ & $\begin{array}{l}\text { señala las } \\
\text { conclusiones más } \\
\text { relevantes de } \\
\text { forma organizada, } \\
\text { sintética y } \\
\text { evalúa/analiza sus } \\
\text { consecuencias }\end{array}$ \\
\hline 5 & $\begin{array}{l}\text { Interpreta, } \\
\text { argumentay justifica } \\
\text { la información } \\
\text { presentada }\end{array}$ & no lo realiza & $\begin{array}{l}\text { Lo realiza de } \\
\text { manera errónea }\end{array}$ & $\begin{array}{l}\text { Lo realiza bien } \\
\text { pero de manera } \\
\text { muy limitada }\end{array}$ & $\begin{array}{l}\text { Lo realiza bien } \\
\text { pero incompleto }\end{array}$ & $\begin{array}{l}\text { Lo realiza de } \\
\text { manera correcta }\end{array}$ & $\begin{array}{l}\text { Lo realiza } \\
\text { correctamente, y } \\
\text { enfatizando los } \\
\text { aspectos relevantes }\end{array}$ \\
\hline 6 & $\begin{array}{l}\text { Presentación } \\
\text { estructurada, clara, } \\
\text { coherente y eficaz }\end{array}$ & no cumple ninguna & $\begin{array}{l}\text { parcialmente } \\
\text { estructurada }\end{array}$ & es estructurada & $\begin{array}{l}\text { al menos es } \\
\text { estructurada y clara }\end{array}$ & $\begin{array}{l}\text { al menos es } \\
\text { estructurada, clara } \\
\text { y coherente }\end{array}$ & $\begin{array}{l}\text { es estructurada, } \\
\text { clara, coherente y } \\
\text { eficaz }\end{array}$ \\
\hline 7 & $\begin{array}{l}\text { Utiliza un lenguaje } \\
\text { técnico adecuado }\end{array}$ & nunca & $\begin{array}{l}\text { 1o usa con errores } \\
\text { reiterados }\end{array}$ & $\begin{array}{l}\text { lo usa con algún } \\
\text { error }\end{array}$ & $\begin{array}{l}\text { lo utiliza } \\
\text { ocasionalmente }\end{array}$ & $\begin{array}{l}\text { lo utiliza habitual y } \\
\text { adecuadamente }\end{array}$ & $\begin{array}{l}\text { lo utiliza habitual y } \\
\text { adecuadamente, e } \\
\text { introduce nuevos } \\
\text { conceptos }\end{array}$ \\
\hline 8 & $\begin{array}{l}\text { Utiliza los recursos } \\
\text { disponibles para una } \\
\text { comunicación más } \\
\text { eficiente }\end{array}$ & $\begin{array}{l}\text { no utiliza los } \\
\text { recursos } \\
\text { disponibles }\end{array}$ & $\begin{array}{l}\text { utiliza los recursos } \\
\text { de forma } \\
\text { inadecuada }\end{array}$ & $\begin{array}{l}\text { utiliza los recursos } \\
\text { pero no para } \\
\text { clarificar ideas }\end{array}$ & $\begin{array}{l}\text { utiliza los recursos } \\
\text { para clarificar las } \\
\text { ideas principales }\end{array}$ & $\begin{array}{l}\text { utiliza los recursos } \\
\text { para clarificar las } \\
\text { ideas de forma } \\
\text { generalizada }\end{array}$ & $\begin{array}{l}\text { utiliza los recursos } \\
\text { disponiblese } \\
\text { introduce nuevos } \\
\text { para clarificar las } \\
\text { ideas de forma } \\
\text { generalizada }\end{array}$ \\
\hline 9 & $\begin{array}{l}\text { La presentaciónse } \\
\text { ajusta al tiempo } \\
\text { disponible }\end{array}$ & $\begin{array}{l}\text { Ningún control } \\
\text { temporal de la } \\
\text { extensión parcial y } \\
\text { total }\end{array}$ & $\begin{array}{l}\text { Se excede o le } \\
\text { sobra demasiado } \\
\text { tiempo }\end{array}$ & $\begin{array}{l}\text { Se ajusta al tiempo } \\
\text { disponible de } \\
\text { forma aproximada }\end{array}$ & $\begin{array}{l}\text { Se ajusta al tiempo } \\
\text { disponible }\end{array}$ & $\begin{array}{l}\text { Se ajusta al tiempo } \\
\text { disponible, dedica } \\
\text { el tiempo } \\
\text { apropiado en cada } \\
\text { parte }\end{array}$ & $\begin{array}{l}\text { Se ajusta al tiempo } \\
\text { disponible, dedica } \\
\text { el tiempo } \\
\text { apropiado en cada } \\
\text { parte y se } \\
\text { reorganiza en caso } \\
\text { necesario }\end{array}$ \\
\hline 10 & $\begin{array}{l}\text { Dicción clara, sin } \\
\text { muletillas, tono } \\
\text { adecuado, postura } \\
\text { corporal adecuada y } \\
\text { contacto visual }\end{array}$ & no cumple ninguna & cumple 1 & $\begin{array}{l}\text { Dicción clara y } \\
\text { tono monótono }\end{array}$ & $\begin{array}{l}\text { Dicción clara y } \\
\text { postura corporal }\end{array}$ & $\begin{array}{l}\text { Dicción clara, } \\
\text { postura corporal y } \\
\text { tono adecuado }\end{array}$ & $\begin{array}{l}\text { Dicción clara, } \\
\text { postura corporal, } \\
\text { tono adecuado y } \\
\text { contacto visual }\end{array}$ \\
\hline 11 & $\begin{array}{l}\text { Analiza, valora y } \\
\text { responde a las } \\
\text { preguntas que se } \\
\text { formulan }\end{array}$ & $\begin{array}{l}\text { No cumple } \\
\text { ninguna }\end{array}$ & $\begin{array}{l}\text { Analiza, pero ni } \\
\text { valora ni responde }\end{array}$ & $\begin{array}{l}\text { Analiza y valora, } \\
\text { pero no responde }\end{array}$ & $\begin{array}{l}\text { Analiza, valora y } \\
\text { responde de } \\
\text { manera limitada, } \\
\text { con ayuda del } \\
\text { profesor }\end{array}$ & $\begin{array}{l}\text { Analiza, valora y } \\
\text { responde con } \\
\text { enfoque propio }\end{array}$ & $\begin{array}{l}\text { Analiza, valora y } \\
\text { responde con } \\
\text { enfoque propio, y } \\
\text { plantea cuestiones } \\
\text { relacionadas }\end{array}$ \\
\hline
\end{tabular}

CALIFICACIÓN:

\begin{tabular}{|c|c|c|c|c|c|c|c|c|c|}
\cline { 2 - 8 } \multicolumn{1}{c|}{ Ítem 1-4 } & Ítem 5 & Ítem 6 & Ítem 7 & Ítem 8 & Ítem 9 & Ítem 10 & Ítem 11 & TOTAL \\
\hline \multirow{2}{*}{ Puntuación } & & & & & & & & & \\
\hline
\end{tabular}

Tabla 6. Rúbrica para la evaluación de la competencia en Comunicación Efectiva oral

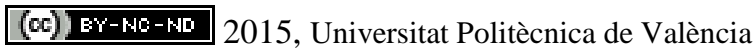

Congreso In-Red (2015) 
Desarrollo de estrategias de evaluación de competencias transversales en asignaturas de ingeniería mecánica y de materiales

\section{Resultados}

En cada asignatura se han aplicado los resultados del proyecto de innovación adaptándolos a su metodología de enseñanza-aprendizaje y a su contenido. Se presenta a continuación la actividad de evaluación desarrollada en una de las asignaturas, a modo de ejemplo. Se trata de la asignatura de Dinámica de Sistemas Multicuerpo ( $3^{\circ}$ Grado en Ingeniería Mecánica), en su parte práctica. Se ha propuesto una actividad complementaria, que se ha incluido de forma ponderada en la nota final de esta parte. La actividad pretende evaluar tanto la destreza adquirida por los estudiantes en el uso de un software de simulación (competencia específica), como su capacidad para aplicar el pensamiento práctico (competencia transversal). La herramienta desarrollada para su evaluación sirve además como guía para que los estudiantes puedan mejorar su trabajo.

En el desarrollo de la asignatura, los estudiantes aprenden a modelar y simular mecanismos reales mediante la aplicación informática de simulación dinámica, ADAMS/View. Durante una serie de ejercicios guiados se modela un motor de combustión interna alternativo de 3 cilindros en línea a partir de un modelo CAD de un motor monocilíndrico (Figura 1).

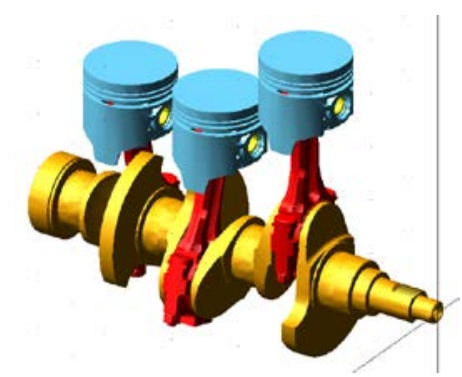

Fig. 1. Modelo en ADAMS/View de un motor de combustión interna alternativo de 3 cilindros en línea

La actividad de evaluación planteada consiste en la redacción de unas instrucciones de montaje de un motor de 4 cilindros en V (Figura 2). Con este trabajo se pretende evaluar la competencia en Aplicación y Pensamiento Práctico ya que para construir el modelo final no existe una única secuencia de pasos, sino que, utilizando los comandos de edición y gestión de entidades del programa ADAMS/View, se puede crear el modelo de manera más o menos rápida y con un número de pasos mayor o menor. Estas diferentes vías son aplicables tanto a la copia y reubicación de elementos físicos del modelo como a la copia y reprogramación de las funciones de las fuerzas en los pistones. 
Javier Carballeira Morado, José Martínez Casas, Óscar Sahuquillo Navarro, Águeda Sonseca Olalla, Francisco David Denia Guzmán, Josep Lluís Suñer Martínez, Paloma Vila Tortosa, Juan José Ródenas García y Onofre Marco Alacid

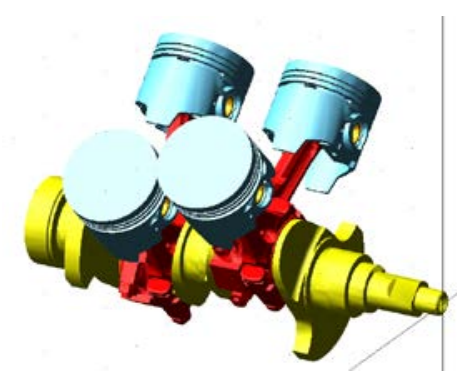

Fig. 2. Modelo en ADAMS/View de un motor de combustión interna alternativo de 4 cilindros en $V$

Además, para la creación del motor de 4 cilindros en $\mathrm{V}$, es necesaria la incorporación de elementos nuevos, debido al alargamiento del asiento de las muñequillas del cigüeñal, ya que se calan dos bielas por muñequilla. El modo en que realizan esta modificación se deja por completo a los estudiantes, que pueden hacerla mediante los elementos propios del programa de simulación o con programas externos de CAD, que permiten trabajar con los modelos originales de las piezas.

Dado que en una vez finalizado el modelo del motor policilíndrico puede ser difícil o imposible detectar la secuencia de pasos que han llevado a ese modelo, se pide una memoria del trabajo bajo la forma de un manual de instrucciones, con un formato similar al utilizado por los profesores de la asignatura en sus clases, de manera que cualquier persona con conocimentos del programa y partiendo de la misma situación inicial sea capaz de llegar al mismo modelo que el realizado por los estudiantes. En esas instrucciones se comprobará si los estudiantes alcanzan los resultados de aprendizaje previstos. Para ello se pasará una lista de control a las memorias de los trabajos cuyos ítems corresponden a los de la competencia transversal, pero se han redactado según las características propias de la asignatura. En la Tabla 7 se muestra esta lista, adaptada de la Tabla 4.

\section{Conclusiones}

A lo largo del proyecto se han planteado diferentes instrumentos de evaluación adaptados a cada metodología y se ha experimentado su uso para anticipar dificultades en su implementación en próximos cursos. Se ha comprobado que los estudiantes de Máster puntúan más alto que los de Grado, en general, y que presentan resultados de aprendizaje en concordancia con su nivel académico en ambos casos, lo que valida la eficacia de los instrumentos desarrollados. La experiencia obtenida ha impulsado a los participantes a proponer nuevas metodologías de evaluación que permiten valorar tanto las competencias específicas, como alguna de las competencias transversales, y que también sirven a los estudiantes para mejorar su aprendizaje.

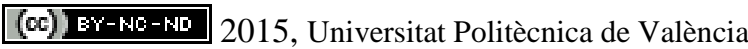


Desarrollo de estrategias de evaluación de competencias transversales en asignaturas de ingeniería mecánica y de materiales

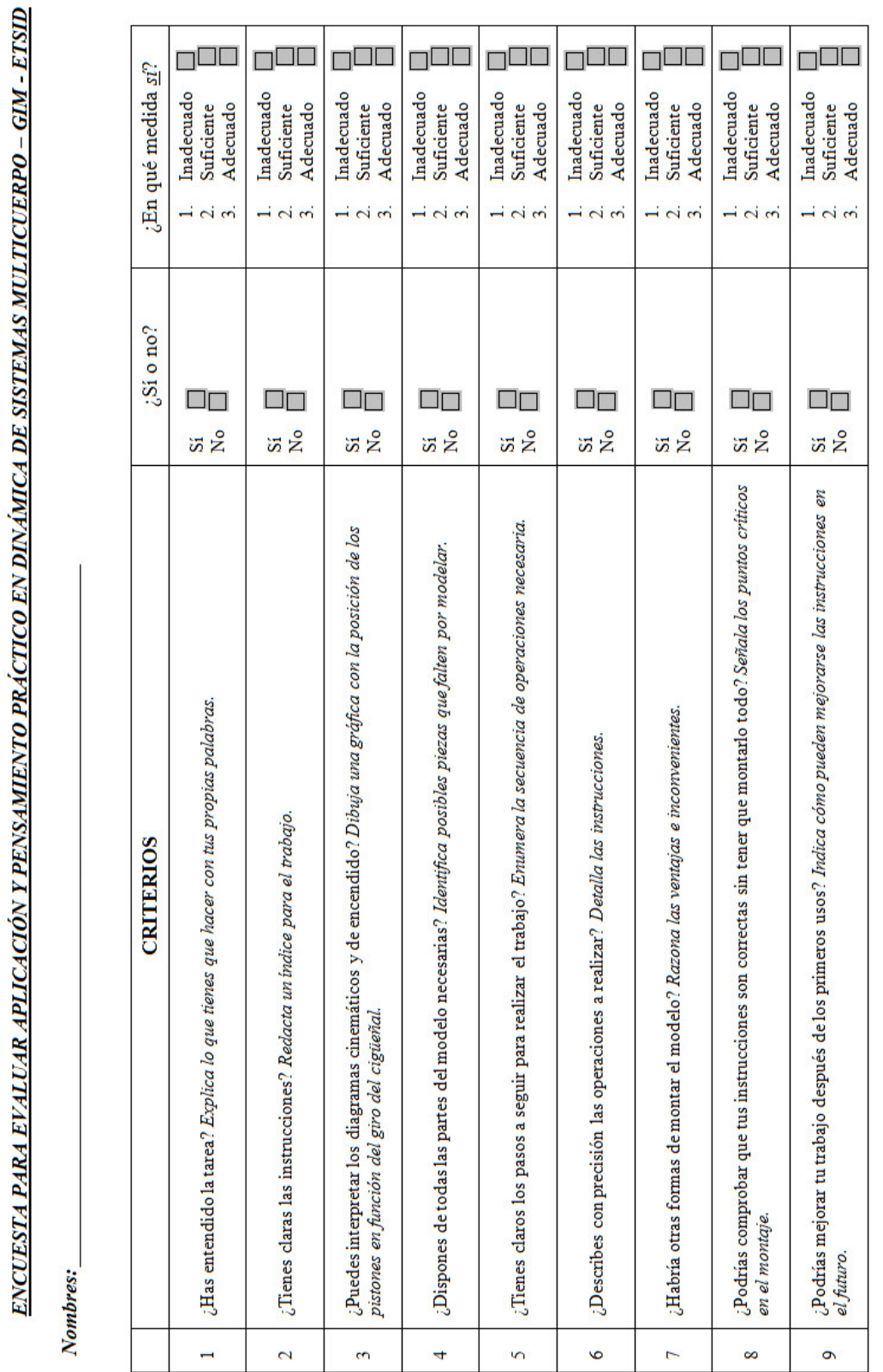

Tabla 7. Lista de control para la evaluación de la competencia en Aplicación y Pensamiento Práctico en la asignatura de Dinámica de Sistemas Multicuerpo, $3^{\circ}$ Grado en Ingeniería Mecánica - ETSID.

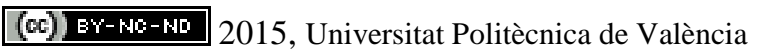


Javier Carballeira Morado, José Martínez Casas, Óscar Sahuquillo Navarro, Águeda Sonseca Olalla, Francisco David Denia Guzmán, Josep Lluís Suñer Martínez, Paloma Vila Tortosa, Juan José Ródenas García y Onofre Marco Alacid

Por último, los autores quieren agradecer la ayuda económica y el apoyo institucional recibidos de la Universitat Politècnica de València a través del proyecto PIME/2014/A/012/B.

\section{Referencias}

ANDREWS, J., y HIGSON, H. (2008). “Graduate employability, 'Soft skills' versus 'Hard' business knowledge: A european study” en Higher Education in Europe, vol. 33, p. 411-422.

DEUSTO (2014). Approaches to teaching, learning and assessment in competences based degree programmes. <http://www.unideusto.org/tuningeu/teaching-learning-a-assessment.html> [Consulta: 22 de julio de 2014]

ENTWISTLE, N. J., y PETERSON, E. R. (2004). “Conceptions of learning and knowledge in higher education: Relationships with study behaviour and influences of learning environments" en International Journal of Educational Research, vol. 41, p. 407-428.

MCDOWELL L., WAKELIN D., MONTGOMERY C. \& KING S. (2011). "Does assessment for learning make a difference? The development of a questionnaire to explore the student response" en Assessment \& Evaluation in Higher Education, vol. 36, issue 7, p. 749-765.

MINISTERIO DE EDUCACIÓN, CULTURA Y DEPORTE, MECD (2002). Marco Común Europeo de Referencia para las Lenguas: Aprendizaje, Enseñanza, Evaluación. $<$ http://cvc.cervantes.es/ensenanza/biblioteca_ele/marco/cvc_mer.pdf> [Consulta: 8 de septiembre de 2014]

MURIAS, P., DE MIGUEL, J. C., y RODRÍGUEZ, D. (2007). “A composite indicator for university quality assesment: The case of Spanish higher education system” en Social Indicators research, vol. 89, p. $129-146$.

POZO MUNICIO, J.I., y PEREZ ECHEVARRIA, M.P (2009). Psicología del aprendizaje universitario. Madrid: Morata.

RIECKMANN, M. (2012). "Future-oriented higher education: Which key competencies should be fostered through university teaching and learning?” en Futures, vol. 44, p. 127-135.

SURSOCK, A. y SMIDT, H. (2010). Trends 2010: A decade of change in european higher education. Brussels: European University Association.

$<$ http://www.eua.be/fileadmin/user_upload/files/publications/eua_trends_2010.pdf> [Consulta: 22 de julio de 2014]

UNIVERSITAT POLITÈCNICA DE VALÈNCIA, UPV (2014). Competencias Transversales.

$<$ http://competencias.webs.upv.es/wp/> [Consulta: 22 de julio de 2014]

(c)) EY-NC-ND 2015, Universitat Politècnica de València

Congreso In-Red (2015) 